\title{
Interseccionalidades y desigualdadesde sexo-género en la producción académica universitaria: el caso de una universidad pública en México
}

\author{
Intersectionalities and gender-sex inequalities in university \\ academic production: the case of a public university in Mexico
}

Dra. Teodora Hurtado $S A A^{*}$

\section{Resumen}

Evaluamos la naturaleza actual y las condiciones del trabajo académico en la Universidad de Guanajuato (UGTO), en el caso de las personas racializadas. Partimos del supuesto de que existen patrones de desigualdad al interior de los centros educativos de nivel superior, que se manifiesta en un orden jerárquico para la designación de los puestos y para la distribución de las cargas de trabajo con base en las interseccionalidades de género, raza, clase y nacionalidad, entre otras. Dicha imbricación fomenta la producción y reproducción de condiciones de trabajo diferenciadas, de acuerdo con la posesión o no de rasgos identitarios subalternizados, incidiendo aún más en la precarización de sus condiciones de empleo, en un ámbito que, pese a su renombre y "prestigio" como lugar de trabajo, en años reciente ha perdido "privilegios" y "seguridad", derivado de la reducción de presupuestos para la educación pública, de las reformas laborales y de la asignación de cargas administrativas adicionales a la producción de investigación y a la docencia; dando paso a la incertidumbre. Si bien las condiciones actuales de precarización del empleo resultan ser generalizadas en México, la UGTO representa un escenario que refleja la desigual posición sociolaboral de las mujeres, más que los hombres, por sus rasgos sexo-genéricos, étnicas/raciales, apariencia y lugar de origen, independientemente de su desempeño profesional. La perspectiva metodológica responde al enfoque decolonial del feminismo antirracista y a la sociología del trabajo, desde los cuales se recopila la información empírica.

Palabras claves: Interseccionalidades, producción académica, precarización laboral, trabajo universitario, desigualdades

"Profesora e Investigadora del Departamento de Estudios Sociales, División de Ciencias Sociales y Humanidades, Universidad de Guanajuato, Campus León. Correos: teodorahurtado@yahoo.es; teodora6811@gmail.com 


\begin{abstract}
We evaluate the current nature and conditions of academic work at the University of Guanajuato (UGTO), in the case of racialized people. We start from the assumption that there are patterns of inequality within higher education centers, which is manifested in a hierarchical order for the designation of positions and for the distribution of workloads based on the intersectionalities of gender, race, class and nationality, among others. This imbrication encourages the production and reproduction of differentiated working conditions, according to the possession or not of subaltered identity traits, further influencing the precariousness of their employment conditions, in an area that despite its renown and "prestige" as The workplace in recent years has lost "privileges" and "security", derived from the reduction of budgets for public education, labor reforms, and the assignment of additional administrative burdens to the production of research and teaching; giving way to uncertainty. Although the current conditions of precarious employment turn out to be generalized in Mexico, the UGTO represents a scenario that reflects the unequal socio-labor position of women, more than men, due to their gendergender, ethnic / racial traits, appearance and place of employment. origin, regardless of their professional performance. The methodological perspective responds to the decolonial approach of anti-racist feminism and the sociology of work, from which empirical information is collected.
\end{abstract}

Keywords: Intersectionalities, academic production, job insecurity, university work, inequalities.

Fecha de recepción: Mayo 2021

Fecha de aprobación: Diciembre 2021

\title{
Introducción
}

Hasta épocas recientes los estudios socio-antropológicos abordaron las categorías de clase, género y raza en forma separada (Wade, Urrea y Viveros, 2008: 18); siendo en fechas recientes que resurge la preocupación de las ciencias sociales latinoamericanas por las interacciones entre dichas categorías, en consonancia con la persistencia de la "colonialidad del poder y del ser" (Quijano, 2000), "del saber" (Lander, 2000) y "de género" (Lugone, 2008), entendiéndolas como los modernos patrones de poder que vinculan la raza, la clase y al género al control del trabajo, al Estado y a la producción del conocimiento (Mignolo, 2000; Quijano, 2000). El proyecto civilizatorio de la conquista representó, por un lado, la dominación y transformación del modo de vida de las mujeres indígenas y negras, y por el otro, la apropiación de su racionalidad y su sexualización (Lugones,2008: 93). 
Por su parte, las contribuciones del llamado Black Feminismo, del feminismo de color y del feminismo tercermundista, destacan la imposibilidad de separar los mecanismos de opresión de género, de la raza y clase. Otras vertientes, incluyendo la teoría interseccional, destacan la necesidad de vincular adicionalmente otras categorías a la discusión y comprensión de las desigualdades y de la discriminación como formas de dominación. Scott (1986) nos recuerda que históricamente el modo complejo en que un conjunto de ideas, discursos y de prácticas se articularon en torno a la institucionalidad del trabajo, la clase, lo masculino y lo femenino llegaron no solo a naturalizarse, también contribuyeron a determinar nuestra percepción sobre el género, la raza, la etnia y el trabajo como fenómenos sociales que involucran dimensiones materiales, culturales, simbólicas y subjetivas.

El argumento expuesto es que la eficacia de determinadas relaciones sociales de dominación funciona y es más impetuosa cuando, al mismo tiempo, se encadena a las relaciones sociales de producción, es decir, cuando se amalgaman a marcos y soportes sociales de apropiación material e inmaterial de la naturaleza (Kergoat, 2003), de la economía, la política y del conocimiento (Hurtado, 2016). Eso significa que las conexiones entre relaciones sociales de género, clase, raza y de producción también son indisolubles, aunque no se hagan manifiestas a simple vista o se ignoren, provocando que el trabajo en general y el de construcción de conocimiento en particular, como quienes lo realizamos, seamos valorados desde nuestra vinculación con el capital y la ciencia, como desde la posición que ocupamos en la relación producción/trabajo y en el orden social (Hurtado, 2011).

Por su condición de género y de clase, aunado a sus características étnicas/raciales subalterizadas, las mujeres en general y negras (e indígenas) en particular han estado marginalmente incluidas al proceso productivo material e intelectual. Para ellas el trabajo como la producción de conocimiento tiene doble connotación; en principio, el trabajo, remunerado o no, es construido como escenario e instrumento para su dominación (trabajos feminizados, domésticos y de cuidado, empleos mal remunerados, salarios inferiores, cargos de subordinación a la autoridad masculina)y como medio para su emancipación (mayores posibilidades de independencia financiera, autonomía personal, capacidad de toma de decisiones, capacidad organizativa, asociativa y de movilización) (Pfefferkorn, 2007: 55-62). Adicionalmente, en contextos universitarios y de producción de conocimiento, el trabajo equivalentemente representa prestigio, libertad de pensamiento, un espacio para la resistencia y la generación de propuestas epistemológicas que le permiten, por un lado, visibilizar las relaciones de poder opresivas que las vulneran, incluso aún más, en escenario aparentemente "igualitarios" como la academia; y por el otro, les brinda posibilidad de construcción de nuevas genealogías, al interior de las mismas estructuras que fundan y sustentan dichas opresiones, procurándose a sí mismas márgenes restringidos de agenciamientos.

Las mujeres afroamericanas, afrolatinas, indígenas, las mujeres inmigrantes y del tercer mundo proponemos teorías en resistencia (Lander, 2000: 4), frentea las propuestas 
epistemológicas cuyo marco de referencia geocultural e intelectual, en el moderno sistema mundo, valida la creencia de que las ciencias y el conocimiento son productos europeos y norteamericano (Rodríguez, 2016; Lander, 2000). Además, son sistemas culturales construidos desde la visión masculina, heteronormativa, de clase y de raza, donde los hombres constituyen el sujeto de referencia por excelencia y las mujeres, en particular las mujeres racializadas, quedan invisibilizadas o excluidas en su calidad de sujetos y de agentes del conocimiento. También se manifiestan contra el feminismo "blanco/mestizo" y burgués, de mujeres cuyos privilegios están definidos por sus sesgos de clase, raza, nacionalidad y orientación sexual, entre otros. Por ello, pese a que continuamos manteniendo una posición subordinada en diversos ámbitos sociales, económicos, políticos y culturales (incluido el entorno académico o universitario), es gracias al trabajo asalariado que las mujeres etnizadas (o no) hemos podido enfrentar y romper algunas de las barreras que limitan el derecho a la equidad, al trato igualitario, a la construcción de epistemologías alternativa a las formas hegemónicas ${ }^{1}$ de conocer, de saber, de ser y del hacer (Hurtado, 2018).

\section{Nadando contra corriente: por una epistemología para enfrentar el racismo, el sexismo y el clasismo en contextos universitarios}

Como mujer negra de tez oscura, afrodescendiente, extranjera, trabajadora e investigadora en contextos universitarios en México, he decidido abordar desde una experiencia (inter)personal, colectiva y auto etnográfica la problemática de las desigualdades sociolaborales en contextos universitarios, en específico en la Universidad de Guanajuato, para generar conocimiento sobre las disímiles, particulares y/o compartidas condiciones de trabajo, considerando la relevancia del autorreconocimiento etnográfico y de la otredad como modelos interpretativos y de presentación de los datos, de quienes pertenecemos a grupos que por nuestra situación de desventaja, como en el caso de las mujeres, los grupos étnicos/racializados, las minorías sexuales, los más pobres y personas con discapacidad, entre otros, reflejan la necesidad de uso y diseño de propuestas teórico-metodológicas de acercamiento a sus realidades, que hagan referencia a las problemáticas que padecen sin invisibilizarlas, considerarlas inexistentes o poco relevantes. Además de negarles estatus epistémico al interior de las ciencias y no reconocer sus narrativas particulares, como integradas a las narrativas de los sistemas de

\footnotetext{
${ }^{1}$ De acuerdo con Flores-Flores (2007: 261) (en su referencia a Pujal 2002), uno de los aportes más contundentes de la teoría feminista ha sido explicar el modo como el nexo entre ciencia y modernidad está profundamente condicionado por un enfoque androcéntrico. Un punto de vista parcial y masculino, que pretende ser un conocimiento neutral, objetivo y aséptico. En su esencia androcéntrica las consecuencias de este enfoque se reflejan en la ausencia de datos y teorías que, desde las propuestas clásicas a la actualidad, hagan referencias a las mujeres, sin el predominio de un lenguaje sexista, sin que las anulen o las invisibilicen. Además, de negarse a dialogar con las teorías feministas o de negarles estatus epistémico al interior de las ciencias y de no reconocer las "historias locales", las narrativas etnias/raciales, genéricas, de clase y demás, particulares.
} 
valores socioculturales y a los criterios cognitivos, apreciativos y morales que los integran (Parsons 2013).

La auto etnografía, de acuerdo con Ellis, Adams y Bochner (2019: 29), se presenta entonces como una metodología cualitativa que tiene como distintivo partir de lo individual, para, desde esa ubicación y conocimiento situado, comprender el contexto espaciotemporal en el que viven y desenvuelven los individuos y grupos, en el sistema de relaciones sociales. La autobiografía también debe mostrar nuevas perspectivas de las experiencias buscando y llenando el "vacío" que existe entre historias relacionadas (Ellis, Adams y Bochner, 2019), contribuyendo a la elaboración de las narrativas y a la descripción de la complejidad que subyace en la posición sociolaboral y, como en el caso que nos compete, en las implicaciones de la figura de profesora e investigadora en la Universidad de Guanajuato, cuando se poseen o no características subalterizadas.

Mi preocupación fundamentalmente se dirige hacia la necesidad percibida de comprender y desarrollar un enfoque amplio o alternativo de trabajo y de sujeto laboral, que me permita dar cuenta de las interseccionalidades de género, etnia/raza, clase, lugar de origen e incluso patrones de belleza y apariencia estética que se afrontan en el entorno laboral en general y académico en particular (en universidades de Latinoamérica y en otras regiones del mundo) ${ }^{2}$, tomando como referencia la perspectiva etnometodológica para el acercamiento a los datos y al estudio de los casos. Asimismo, recurro al método biográfico para la reconstrucción de las trayectorias sociolaborales y el diseño de un perfil tipológico que, al hablar en primera persona del singular y del plural o en "voz en off", me permita recrear la realidad de los docentes universitarios en esta alma mater; no solo como espacio de trabajo intelectual, sino también como escenario de relaciones sociales cimentadas, paradójicamente, sobre la base de "combatir", a través de la producción de conocimiento, las estructuras sociales de poder y de dominación, las desigualdades en las que se sustentan dichas estructuras y que imponen opresiones imbricadas y simultáneas, muchas veces invisibilizadas y/o naturalizadas, como las discrepancias que preexisten en la interseccionalidad entre racismo, clasismo, discriminación, sexismo por el hecho de ser mujer, de ser negra, de ser extranjera y de ser considerada como "atractiva" antes que trabajadora intelectual, en un contexto donde dichas cualidades pueden y hacen la diferencia entre alcanzar movilidad laboral o no al interior de un institución educativa de nivel superior.

En otros términos, en un escenario de producción académica donde hasta recientemente era casi la única persona negra o afrodescendiente de tez oscura, en la sede en que trabajo, el exotismo que representa mi presencia y el de otras académicas afrodescendientes está al orden del día. En ese contexto, aunque no se pretenda llamar la atención ni generar expectativas, más allá del estricto cumplimiento de las funciones

${ }^{2}$ El presente artículo responde a los aportes preliminares de una investigación en curso de la cual son responsable, denominada "Adiós a los trabajos protegidos, formales y de prestigio: condiciones de trabajo y precarización laboral en contextos universitarios (o educativos). Análisis de casos, financiada por el CONACYT-Ciencia Básica. 
laborales, el problema es que por la condición racial se asume que puedes fallar, aunque "trabajes como negra para vivir como blanca", y si llegas a triunfar se pretende que actúes como el "buen salvaje" o a través de las interacciones sociales "merecer" la aceptación o aprobación del entorno, es decir, acceder a mayores cargas de trabajo, ceder o compartir los créditos de la producción laboral personal con otros y otras cuyo privilegio de género, raza y/o cargo les coloca en una condición superior. Bajo este esquema equivocarse y/o no compartir estos valores más que un hecho se convierte en un lujo que los sujetos sociales subalterizados no se pueden dar o lo hacen corriendo el riesgo de ser excluidos 0 rechazados.

Para dar cuenta de esta realidad, metodológicamente la presente propuesta se desarrolló con base en un enfoque predominantemente cualitativo, que contempla la recopilación de datos en interacción y convivencia con las personas involucradas, como resultado del trabajo de campo, de la observación y de la autoetnografía realizada al interior de la Universidad de Guanajuato; asimismo, se toma como referencia los relatos de hombres y mujeres que laboran en esta universidad, ya sea como profesores e investigadores de tiempo completo o como docentes de tiempo parcial, en diversos Campus y Divisiones adscritas a dicha alma mater. Sin embargo, además de sus perfiles sociolaborales y académicos, se toma en cuenta sus rasgos identitarios más generales: sexo-género, etnia/raza, clase, lugar de origen, edad y niveles educativos, al igual que sus trayectorias como investigadores, entre otros rasgos relevantes para establecer y analizar las condiciones actuales en las que se construye, practica y se percibe el ejercicio del trabajo universitario, de parte de los agentes implicados, los cuales poseen múltiples rasgos e identidades.

Como nota aclaratoria, la referencia autobiográfica constante o el referirme en primera persona a lo largo del documento, en algunos relatos y en varias descripciones responde a la necesidad de resguardar y proteger la identidad e información personal de quienes compartieron confidencias y opiniones para este estudio, tomando en cuenta las implicaciones y resistencias en el contexto laboral al platicar sobre estos temas. En ese sentido, y desde el enfoque decolonial, la autoetmografía me permite, más que estudiar a los otros y otras como sujetos objetos de investigación, generar un proceso dialógico y un conocimiento situado, en términos de las realidades compartidas, a la vez que doy testimonio de las diversas experiencias de opresiones y desigualdades. Rompo así con una lógica de la colonialidad del saber: estudiar la otredad, la opresión y la desigualdad sin percibirse como parte de ello y asumiendo el crédito por una labor académica producida en interacción.

Durante el desarrollo de la investigación, se contó con una base de datos brindada por la misma institución y con las cifras reportadas en las páginas institucionales de acceso a la información pública, donde se presentan registros de empleados universitarios, número de trabajadores y las categorías de contratación, los procesos de 
certificación (tipo $\mathrm{SNI}^{3}$ y $\mathrm{PRODEP}^{4}$ ) con los que cuentan los profesionales académicos. Adicionalmente se recopila información de medios electrónicos e Internet, que hacen referencias a sucesos y noticias sobre diversas problemáticas acaecidas al interior y exterior de la Universidad de Guanajuato, en particular, para ahondar en algunos datos e información y dar cuenta de fenómenos y conflictos en los cuales se ven imbricadas dinámicas laborales y procesos institucionales.

En cuanto a la temporalidad de la información, el trabajo de campo se realizó entre 2018 y 2020, aunque puede hacer referencia a periodos de tiempo anteriores o a testimonios de experiencias acontecidas a lo largo de las trayectorias laborales en la UGTO, tomando como argumentos los relatos descritos y la evidencia recopilada en el desarrollo del trabajo de campo e información aportada por más de 30 entrevistados y entrevistadas en el proceso. Sin embargo, para este documento solo se incorporan algunos aspectos cualitativos, en particular los referidos a la interseccionalidad, a la producción científica y a la apropiación epistemológica del conocimiento interpretados desde el afrofeminismo y el feminismo decolonial como una manera de (re)plantearme mi condición de mujer negra profesora e investigadora.

\section{El espacio de trabajo académico y las construcciones identitarias divergentes}

De acuerdo con la cita de Habermas que hace Dominique Méda (2007: 17), las sociedades actuales hicieron del trabajo no solo el principal medio de transformación de la naturaleza y de subsistencia, también se convirtió en un hecho que ocupa una parte esencial de la vida de los individuos, en particular porque el trabajo se cristalizó en parte de las estructuras que organizan la vida, ordenan lo social y determinan el lugar de los individuos, haciendo de la acción de trabajar, más que una práctica, un "hecho social total" y totalizante (op. cit). En esa trayectoria, con el advenimiento de las sociedades modernas, del capitalismo, de la propiedad privada sobre los medios de producción y de

\footnotetext{
${ }^{3}$ Sistema Nacional de Investigadores. EI SNI fue creado Por Acuerdo Presidencial publicado en el Diario Oficial de la Federación el 26 de julio de 1984, para reconocer la labor de las personas dedicadas a producir conocimiento científico y tecnología. El reconocimiento se otorga a través de la evaluación por pares y consiste en otorgar el nombramiento de investigador nacional. Esta distinción simboliza la calidad y prestigio de las contribuciones científicas. En paralelo al nombramiento se otorgan estímulos económicos cuyo monto varía con el nivel asignado https://www.conacyt.gob.mx/Sistema-nacional-de-

investigadores.html\#: :text=El\%20Sistema\%20Nacional\%20de\%20Investigadores,producir\%20con ocimiento\%20cient\%C3\%ADfico\%20y\%20tecnolog\%C3\%ADa..

${ }^{4}$ Es el Programa para el Desarrollo Profesional Docente (PRODEP), que tiene como objetivo contribuir para que el personal docente y personal con funciones de dirección, de supervisión, de asesoría técnico-pedagógica y cuerpos académicos accedan y/o concluyan programas de formación, actualización académica, capacitación y/o proyectos de investigación para fortalecer el perfil necesario para el desempeño de sus funciones. http://www.itssnp.edu.mx/downloads/Contraloria_Social/PRODEP.pdf
} 
la especialización de la fuerza de trabajo, sobreviene el contraste entre el trabajo manual y el trabajo intelectual; el primero percibido como una actividad realizada por las clases oprimidas (esclavos, siervos y obreros), mientras que el segundo (ciencia, arte y cultura) se le ha relacionado con las clases dominantes o privilegiadas (Neffa, 2003).

Visto en perspectiva, la centralidad de trabajo en la vida de los individuos reside en la premisa de que las sociedades organizan las relaciones sociales en torno al trabajo y el capital (Méda, 2007:28);por tanto, resulta evidente que cuando afirmamos que el trabajo es condición para ser considerados miembros activos de la sociedad, es porque las personas carentes de trabajo y/o de riqueza se encuentran al mismo tiempo desposeídas y excluidas social, económica y políticamente (Hurtado 2016:97-98). En otros términos, paralas sociedades modernas el trabajo cumple funciones indispensables como factor de cohesión e interacción social, como lo evidencian los contactos que se fundan dentro del ámbito laboral y se extienden más allá del interior y del exterior del entorno familiar; del mismo modo, favorece el ejercicio de la ciudadanía plena, proporciona identidad social e impulsa a la acción.

Gracias al trabajo, no solamente obtenemos y disfrutamos de los medios para vivir; de igual manera, el trabajo capacita, posibilita y otorga las herramientas para mejorar. Así, el trabajo aparece como la energía, propiedad de los sujetos que les permite volverse cultural y socialmente valiosos para las instituciones. Sin embargo, pese a la centralidad del trabajo en la sociedad y en la vida de las personas, el problema está en que la noción de sujeto laboral no es homogénea y tampoco precisa de ser democrática o igualitaria.

En este contexto, el trabajo puede considerarse como fuente de realización personal o, al contrario, puede calificarse como limitante del desarrollo personal y social. Reconociendo a su vez que existen condiciones en el proceso de trabajo: el tipo de empleo, su carácter formal o informal, las condiciones de trabajo, el clima organizacional, la jerarquización, la división sexual de las actividades, el estatus que se ocupa en la pirámide ocupacional y la cultura laboral, que inciden para que la vida laboral se experimente de manera desigual, de acuerdo con las interseccionalidades sexo-genérica, étnicas/raciales, de clase, tipo de contratación, política de la apariencia, etc. (Hurtado, 2018). Dicho contraste, entre trabajo intelectual y manual, por ejemplo, no solo se ha acentuado, también impone condiciones de trabajo desiguales, como resultado de los fenómenos de estratificación social, los cuales de acuerdo con García (1999) sitúan a las personas jerárquicamente en los distintos sistemas ocupacionales. En la sociedad actual, el criterio fundamental es la habilidad de ganar dinero (García 1999: 138).

Se adquiere por ende un estatus a través de la profesión que desempeñamos, no solamente desde el punto de vista económico, sino que cada profesión y cargo sociolaboral otorga mayor o menor prestigio social, entre las que tradicionalmente se encuentra el trabajo intelectual. Además, también la profesión da pauta a una cierta posición de poder, colocando a personas con puestos de representatividad (líderes de partidos políticos, gobernantes y representantes públicos, directivos de instituciones 
académicas o asociaciones de profesionales, etc.) en una posición social de mayor relevancia, frente a personas con otras profesiones, cargos y/o capacidad de toma de decisión (García, 1999).

Desde la lógica de Amartya Sen (2000) y de Martha Nussbaum (2012), el tener un trabajo gratificante es uno de los aspectos fundamentales para alcanzar el tipo de vida al que aspiramos llegar. Concretamente, establecen que una preocupación fundamental para los individuos es ser capaces de vivir el tipo de vida al que aspiran, incluyendo en este ideal tener un trabajo gratificante, puesto que funge como un posibilitador para que las personas puedan tener una vida física y mental saludable, contribuyendo a una visión integral de desarrollo que contemple tanto la dimensión material de la vida como el bienestar y las capacidades de las personas (Gutiérrez y González, 2010). Sin embargo, la visión hegemónica se ha concentrado más en la dimensión económica y política y dejado de lado aspectos sociales y culturales. Bajo esta lógica, la vida moderna ha venido acompañada con trabajo de contratos a corto plazo, flexibilización y precarización de las condiciones de empleo. Entre otras circunstancias que debilitan la forma tradicional en la que se percibía la carrera profesional, y convirtiendo a las empresas o a las instituciones educativas en organizaciones horizontales y flexibles. Esto a su vez ha impactado en los vínculos sociales, afectando directamente las vidas emocionales de las personas (Sennett, 2000). Bajo este impulso de formas flexibles de organización y nuevas tecnologías se sustenta aquello que tradicionalmente llamamos progreso, caracterizado por el incremento de la productividad y competencia global, aunque con patrones de desigualdad cada vez más profundos y marcados (Castells, 2002).

Como acontece con todo fenómeno social, las visiones e interpretaciones sobre el trabajo también son construidas de manera desigual e interseccional. De hecho, como lo presenta Méda (2007:19) la contribución de la Grecia antigua a la noción de trabajo fue, por una parte, relacionarlo con las formas de servidumbre, la esclavitud y la necesidad de producir a partir de la explotación laboral de la fuerza de trabajo remunerada o no, y por el otro, articularlo con dependencia económica y estatus social. Bajo esta visión, tanto el ejercicio del trabajo como quienes lo realizaban se situaban en la condición de subordinados, caso de los trabajos feminizados y la fuerza de trabajo esclavizada o en condiciones de servidumbre, ya que estos desarrollan las actividades de menor jerarquía a la vez que pertenecen a una clase social subalterizada; por tanto, se demanda de parte de ellos sumisión a la necesidad u obligación de participar en el proceso de reproducción de las condiciones materiales de existencia para otros, pero no se les permite disponer de las mismas libertades para beneficiarse de las bondades de dicho quehacer en igualdad de condiciones.

La dinámica social hace así que el trabajo considerado femenino o que desarrollan las mujeres se encuentre devaluado frente al trabajo de los hombres, mientras que la dimensión racial obra para que simultáneamente las actividades emprendidas por las personas negras (e indígenas) se conciban como inferiores. Dicha condición de subordinación actúa para que la producción de conocimiento artístico e intelectual, en el 
caso de las personas negras, se presente como carente de genialidad, entendida esta como la capacidad para concebir y elaborar cosas o ideas admirables, e incluso para realizar actividades de forma novedosa, creativa e intelectual, en un sistema donde este valor es atribuido a una entidad simbólica universalizada: "invento del hombre blanco occidental", es una frase acuñada incluso en el argot de académicos y académicas mestizados en México, que hace referencia una entidad por lo demás androcéntrica, caucásica, euro y norcéntrica, que coloca en los márgenes de la periferia intelectual la producción cultural de las personas afro e indígena en general y de las mujeres negras e indígenas en particular. Igualmente, niega la genialidad de los territorios y cuerpos racializados del sur (es decir de América Latina, de África y de Oriente) ${ }^{5}$.

En contextos académicos de nivel universitario, por ejemplo, el trabajo es percibido como un tipo de empleo de "prestigio", "privilegiado", "honorable" y "seguro", percepción queen el caso de las personas negras se ve alterada por causa del racismo académico e intelectual (Castillo y Moré, 2016). En la Universidad de Guanajuato, las condiciones bajo las cuales se ejercen las actividades de investigación, docencia y extensión obligan a que las mujeres tengan jornadas de trabajo más largas y cargas más exhaustivas, ello implica desarrollar un conjunto de actividades laborales invisibilizadas: mayor disposición para la formación de estudiantes, para el trabajo colegiado, para las tutorías, para la actualización y capacitación docente, incluso para el trabajo emocional de cuidado e interacción social, lo que genera mayor valor agregado y rentabilidad institucional, gracias a la plusvalía emocional y a la autoexplotación laboral que el trabajador (en particular las trabajadoras) hace de su fuerza de trabajo, aunque no obtenga o reciba menor reconocimiento o mínimas compensaciones adicionales a su salario por dicha asignación. Estas son en sí mismas cargas o trabajos invisibilizados, que además de no reconocerse, implican extender la experiencia de trabajo más de las 40 horas establecidas, al punto de que la carga laboral se incrementa la mitad o el doble,

${ }^{5}$ Los patrones de relaciones sociolaborales observados en el caso de estudio responden a los sistemas de relaciones sociales producto de la colonialidad/modernidad, descritos por autores como Quijano, Dussel, Minolo, Castro-Gómez, Grosfoguel como patrones decolonialidad del poder y del ser. Estos autores y la producción teórica de la colonialidad/modernidad parten del supuesto de que la división internacional del trabajo entre centros y periferias, así como la jerarquización étnico-racial de las poblaciones, formada durante varios siglos de expansión colonial europea, no se transformó significativamente con el fin del colonialismo y la formación de los Estados-nación en la periferia. Más bien lo que se produjo y a lo que asistimos es a una transición del colonialismo moderno a la colonialidad global, proceso que ha transformado las formas de dominación desplegadas por la modernidad, pero mantuvo intactas las estructuras de relaciones entre centroperiferia, de explotación laboral, los procesos de racialización de los grupos subalterizados y de las mujeres a escala mundial (Castro-Gómez y Grosfoguel 2007: 13)

${ }^{6}$ Colonialidad Discursiva (ChandraMohanty, 1988), Violencia Epistémica (GayatriSpivaK 1999) y Epistemicidios (Boaventura De Sousa, 2018) son conceptos que desde el pensamiento decolonial y desde el femenino poscolonial se han elaborado, para describir las formas de apropiación del conocimiento e invisibilidad de la producción intelectual y cultural de los territorios y de las poblaciones colonizadas desde la colonialidad del saber y del ser. 
sobre todo, cuando hablamos de ser consideradas parte de un grupo subordinado: como mujer, negra (o indígena), soltera o sin responsabilidades familiares.

Apelando a la noción griega que plantea Méda, la servidumbre y la esclavitud moderna como formas de trabajo se arraigan en los contextos laborales, pero en el caso de la academia, este ya no es ejercido desde la necesidad productivista de la fuerza de trabajo, ya sean mujeres y hombres negros (o indígenas), visto como tales (ayudantes o subordinados). Al contrario, Adolfo Vásquez Rocca (2007: 12), en su estudio sobre la sociedad de la transparencia, autoexplotación neoliberal y psicopolítica, argumenta que en nuestra época, el trabajo se presenta en forma de libertad y autorrealización, es decir, que mientras procuramos alcanzar el reconocimiento y liberarnos de las condiciones de subordinación, entramos en una lógica de la (auto)explotación: me exploto a mí misma o mismo pero creo que me realizo, lo que induce a que la sensación de alienación se suprima y, por consiguiente, se facilite la optimización de la productividad de la fuerza de trabajo. Esta autoexplotación es más eficaz y poderosa que la explotación realizada por el capital sobre la mano de obra (como lo refiere el marxismo), porque va acompañada de un sentimiento de libertad: libertad de cátedra, libertad de investigación, libertad de producción, libertad para la generación de conocimiento. Se construye, entonces, un trabajador doblemente explotado, porque se explota a sí mismo y también es explotado por la empresa. Pero contrario a lo que Vásquez Rocca (2007: 12) reporta en su estudio, consideramos que en el caso de los sujetos subalterizados estudiados, más que impedir las formas de resistencia, la sobre explotación representa paradójicamente revolución, constituye una ética del trabajo y de productividad ilimitada como herramientas para enfrentar el racismo, los prejuicios, la discriminación y la exclusión apoyados en la optimización personal y profesional como formas de liberación e independencia del otro ${ }^{7}$. Trabajar más que el otro/otra, producir más que el otro/otra y obtener mejores resultados le permiten alcanzar, por lo menos, una especie de inclusión laboral, pero segmentada a falta de otras posibilidades.

Se hace manifiesta la categorización de los individuos y de los grupos sociales según criterios preestablecidos y validados socialmente, los cuales se revelan como prejuicio y/o estereotipos, desde los cuales se "marcan" a los trabajadores antes y después de contratarlos, en función de su género y de sus rasgos físicos. Según Carrasco (2008), pese a la magnitud de capital humano, académico, técnico e intelectual que los ofertantes de fuerza de obra puedan poseer, se registra una segregación ocupacional de las personas negras discriminadas perceptible con la sobre concentración

\footnotetext{
${ }^{7}$ De acuerdo con Lugone (2008) y con Curiel (2015) la organización social del mundo colonial incluía a las mujeres negras e indígena como objeto de dominación en cuanto a su raza y su sexo. La colonialidad del género, al estudiar la transversalidad de la raza, clase y género, se centraliza en la triple (y en las múltiples) dimensiones de la desigualdad que las mujeres indígenas y negras experimentan, debido a que en cuanto a su raza son invisibilizadas, explotadas en cuanto a su clase, y como mujeres son construidas como inferiores, según el estereotipo del mundo moderno occidental. Esto es parte de la misma producción de la colonialidad del poder, del saber y del ser (Rodríguez, 2016: 148-149).
} 
en pocas y determinadas ramas de actividad, percibido igualmente en su contrastante ausencia de otras y/o de los mandos medios debido a las escasas oportunidad desde movilidad laboral ascendente.

Los datos estadísticos del estudio sobre el impacto de las características étnicoraciales en la desigualdad de oportunidades en México, publicado por Solís, Güémez y Lorenzo (2019:3), son concluyentes: identificarse dentro de una comunidad indígena, negra o mulata, o tener el color de piel más oscuro resultan en probabilidades menores de avanzar en el sistema educativo, progresar en el ámbito laboral o pasar a la parte más alta de la distribución de la riqueza. Y dentro de esta plétora de discriminación, ser mujer indígena es la que genera una marea en contra más fuerte. Pese a las condiciones privilegiadas que puede llegar a ofrecer el entorno universitario como espacio laboral, las personas racializadas entran en una dinámica de autoexplotación, de autoexigencia, de constante optimización de sus capacidades que le permita luchar contra las violencias sutiles e institucionalizadas del racismo, del clasismo, del sexismo, entre otras formas de relaciones de poder y dominación, a la vez que procuran ser concebidas como sujetos laborales constructores de genealogías.

Estos factores me han permitido entender mi propia situación sociolaboral y académica en la Universidad de Guanajuato (al igual que la situación de otras académicas y académicos racializados) como mujer, negra, extranjera de origen colombiano, con una apariencia estética por lo demás llamativa que rompe tanto con los patrones de belleza y raciales locales (de tez oscura, alta, delgada, guapa e inteligente), como con las conductas tradicionalmente esperadas en una mujer (al ser considerada problemática y de carácter fuerte), como en ocasiones me describen (Hurtado 2016: 102). Conforme este contexto sociolaboral, me concibo como mujer afrodescendiente de tez oscura y como académica, antes que definirme por mi apariencia estética y corporal, grado académico, clase y demás; aunque en determinados contextos inicialmente soy percibida como "exótica" y "peligrosa" (Hurtado 2016),o se me cuestiona como alguien cuya presencia molesta por el hecho de llamar mucho la atención, lo que personalmente concibo como una situación de opresión, desde la cual no se cuenta con elementos para objetar mi ejercicio profesional, pero se pone en duda.

Entender estas conexiones, en el caso de las mujeres negras (etnizadas, racializadas, sexualizadas, erotizadas, estigmatizadas por su apariencia estética y corporal) abre la posibilidad de comprender el efecto transversal e interseccional de los sistemas de opresión y las relaciones sociales de poder; asimismo, me permite construir una epistemología de la desigualdad, de la interseccionalidad y de los sistemas de opresión en contextos universitarios. En opinión de Parella (2005:106), pese a que la interseccionalidad afecta las experiencias de vida de todos los seres humanos, tanto en la esfera productiva como en la reproductiva, las mujeres negras e indígenas se sitúan en un escenario de clara desventaja frente a quienes se ubican en la cima del sistema de jerarquización, gracias a los privilegios y beneficios que les merece su situación de ser 
mujeres (y hombres), blancas/mestizas. Como igualmente lo evidencian Solís, Güémez y Lorenzo (2019) en el caso mexicano.

En este sentido, los vectores de opresión y privilegio evidencian que las condiciones de desigualdad son relacionales y contextuales, por cuanto las categorías hombre/mujer, blanco/negro, bello/feo, sujeto de trabajo intelectual o no, sólo tienen significado cuando se ponen en contraposición una a la otra. Por tanto, la opresión de una mujer negra en una sociedad racista y sexista se presenta como si se tratara de una carga adicional, cuando en realidad es una carga diferente; porque ser mujer de "razanegra" o "indígena" significa ser mujer y ser negra, o ser mujer y ser indígena, y en el contexto universitario significa construirse desde otras realidades y epistemologías, una cuya tarea sea hablar de las desigualdades y de los sistemas de opresión por raza, género, clase y de producción intelectual al interior de la academia, una en que el hecho de investigar y formar capital humano se transmuta de producción de conocimiento, docencia y militancia en un acto de transformación revolucionaria o de resistencia intelectual.

Del mismo modo, en la academia hay mujeres (u hombres) que "tienen todo" o, en otros términos, se benefician de los privilegios que le otorga su pertenencia a una categoría étnica/racial, de clase y orientación sexual socialmente más valorada, en comparación con otras que no tienen estos mismos vectores de privilegio y, por tanto, su condición social y ocupacional es más precaria. En este sentido, carece de claridad interpretativa referirme a un sujeto femenino genérico, cuando la realidad evidencia que lo femenino es una construcción social intersubjetiva y una categoría internamente fragmentada (Hurtado 2016: 102). Al respecto Teresita De Barbieri, desde el año de 1993 cuestiona el hecho de que la clase y el estatus social, desde la emergencia de los movimientos feministas y de los estudios sobre las mujeres en la región de América Latina, no solo ponen en evidencia las relaciones de desigualdad entre mujeres, y cómo estas desigualdades tienen lugar en diversos ámbitos de la vida social, también dio cuenta y puso al descubierto las tramas de relaciones sociales en función del ejercicio del poder y la autoridad entre mujeres, que a pesar de compartir la misma posición de clase y situación de subordinación masculina, pueden ejercer poder y dominación sobre aquellas mujeres a quienes perciben o consideran como inferiores (o problemáticas para el sistema por su condición de clase y/o de raza, ocupación, nacionalidad, orientación, activismo y producción intelectual, etc.) advirtiendo que algunas mujeres pueden gozar de poder sobre las otras. Más aun, en contextos de alta dominación masculina (como en el caso de la UGTO donde el 95\% de los cargos de autoridad están en cabeza de los hombres), ésta puede tener como agente dominador a ciertas y determinadas mujeres, al investirlas de autoridad. Ello significa que el sistema crea zonas de incertidumbre, divide a las mujeres, impide alianzas y se legitima asimismo como escenario para la dominación (De Barbieri, 1993: 157).

El feminismo occidental tomó como referente a la mujer blanca, de clase media y de países industrializados, mientras que, sin un posicionamiento crítico, algunas 
corrientes del feminismo en la región y en la Universidad de Guanajuato han adoptado estas posturas como propias, excluyendo del razonamiento intelectual y organizativo a las otras mujeres por sus categorías de diferenciación: a las mujeres negras ("porque están sexualizadas"), a las mujeres indígenas ("porque están sometidas por la cultura"), a las mujeres con orientaciones e identidades sexuales diversas y trans ("porque no son mujeres").

Más allá de tratarse de una presencia invisibilizada y subvalorada, cuando esta participación en el ámbito universitario se analiza en función de las intersecciones entre las características raciales, sexuales y étnicas, las mujeres (y los hombres) indígenas y afrodescendientes presentan las condiciones más complejas (Hill Collins, 2000). Como lo señala Hurtado (2016: 94), las relaciones sociales opresivas por condición de sexogénero y la división sexual del trabajo les otorgan a las mujeres negras (e indígenas)una posición social específica, una condición de subordinación desde la cual ellas contribuyen a reproducir la fuerza laboral, a producir capital y a construir intelectualidad, pero no se les considera como parte trascendente en ello o las ocupaciones, como la producción científica, que realizan son concebidas como de menor valor agregado frente a la masculina/femenina blanca/mestiza.

\section{Pos-colonialidad e Interseccionalidad del ser y del saber en la academia: un análisis de resultados}

Por su calidad y condiciones de empleo, el trabajo académico desarrollado en el contexto de las universidades no había sido considerado causante de sufrimiento laboral o personal, en otras palabras, no se le había considerado una fuente de stress ni de sufrimiento desde el punto de vista socio-ocupacional ni científico. Ello se debía fundamentalmente a las características laborales que tradicionalmente se le asignaba al trabajo intelectual (Llomovatte y Wischnevsky, 1998: 16), de empleos que se distinguían del trabajo físico o manual por su nivel de formación técnica, su dedicación a la producción científica, al arte, a la cultura y a la política; asimismo, se trata de actividades donde predominaba una relación salarial formal, cobijados por un contrato de duración por tiempo indeterminado, con la garantía de estabilidad, declarado o registrado ante la administración del trabajo y el sistema de seguridad social, al realizarse de parte de los trabajadores y de los patrones los aportes correspondientes, lo cual otorgaba una adecuada protección social que beneficiaba también a la familia del trabajador, dado a que aún después de que este como titular falleciera se le otorgaba la pensión o jubilación a sus beneficiarios(Neffa, 2010: 2-3). Garantías que le permitían al trabajador o a la trabajadora y a su parentela cimentar la idea de perdurabilidad, movilidad social y espacial, seguridad, solidez y continuidad de los recursos necesarios para mantener las condiciones materiales de existencia, con base en el empleo.

Sin embargo, el escenario actual de precarización del mercado de trabajo, del empleo y del poder adquisitivo de los asalariados, al igual que las condiciones opresivas 
que experimentan las mujeres negras en el contexto universitario nos han hecho despertar de esa "certidumbre" retrospectiva: pensar que el trabajo en general y el que se efectúa en contextos universitarios, en particular, serían medio de realización personal, plataforma para el desarrollo de la identidad colectiva e individual y garantía del desarrollo tanto social como nacional e individual. En las últimas décadas, cuando las creencias y realidades en torno al trabajo como medio de realización se han transformado (Méda, 1996:688-690), también se han modificado las maneras de entender e imaginar el empleo en general y el empleo académico en particular. Se trata entonces de abordar los significados actuales del trabajo en general y del entorno académico en particular, develar el sexismo, el clasismo y el racismo que los sustentan a partir de otra epistemología: la genealogía afro, interseccional y decolonial.

En este sentido vale destacar el estudio sobre el racismo en México, publicado por Mónica G. Moreno Figueroa en el 2016, quien matiza que, aunque existe una constante negación de este fenómeno sociopolítico, económico y cultural, el racismo en este país existe; por tanto, es importante que esclarezcamos sus manifestaciones y evidenciemos sus consecuencias. No sólo porque rebasa la vida cotidiana y moldea las sensibilidades y las relaciones sociales, principalmente porque el racismo es un precepto que afecta negativamente a las poblaciones negras e indígenas, establece parámetros de inclusión y exclusión, de privilegios y opresiones, con base en juicios y prejuicios históricamente construidos, para la distribución de los derechos y de las injusticias (Moreno, 2016: 92). En la cita que la autora hace del trabajo de Hartman (2007: 129), Moreno asimila el racismo con la "distribución social de la muerte; como una gráfica actuarial, predice quien va a florecer y quien no"8. Por eso, su estudio constituye una interesante disertación que, desde los márgenes de su condición de mujer afromexicana y académica universitaria, contribuye a la integración de una epistemología que plasma y presenta el racismo como un eje central en la organización social, en las experiencias de la vida cotidiana y en el entramado institucional.

Desde su disertación intelectual y académica podemos reconocer que las universidades, como instituciones de producción y construcción de conocimiento o como espacio de trabajo, también fortalecen y fomentan discurso racializados, alimentando un contexto en el que la creciente ideología posracial viene a reforzar y a encubrir el resurgir

\footnotetext{
${ }^{8}$ Previa a la propuesta de la escritora Saidiya Hartman (sobre la distribución social de la muerte en su libro Lose your Mother: A Journey along the Atlantic Slave, publicado en el año de 2007), el filósofo Achille Mbembe desarrolló el concepto de Necropolítica, para describir como algunos estados, grupos de poder e individuos consideran el aniquilamiento físico y/o moral de otros como un derecho: el derecho a matar y a exponer a otros individuos o grupos sociales a la muerte, a la esclavitud o someterlos a diversas formas de violencia. La Necropolítica, por tanto, se refiere a las formas de subyugación, de control social y político como poder de la muerte y a sus víctimas como muertos vivientes, fomentando condiciones de vida precarias para algunos cuerpos e induciéndolos a permanecer en diferentes estados de vida y muerte (particularmente cuerpos racializados, higienizados, torturados, violentados, etc.)
} 
del proyecto étnico del mestizaje. En ese sentido, la doctrina posracial ${ }^{9}$ es entendida comoel supuesto ideológico de superación del racismo y la manifestación de una etapa enla cual el prejuicio racial ya no existe, o ya no es causa de problemáticas sociales como la discriminación, la exclusión y la marginación por condición étnica/racial, dado que serían sustituidas por otras problemáticas vinculadas con el incremento poblacional, el desarrollo industrial y las crisis económicas.

Desde el paradigma epistemológico, las ideologías posraciales representan a todas las "formas de pensamiento, discurso y acción que evaden, deslegitiman y buscan eliminar las diferencias raciales y sus efectos como tema y enfoque del conocimiento académico, de la lucha activista, del debate público y de las políticas estatales" (Da Costa, 2014: 2). La estrategia posracial pretende minimizar la preeminencia de las nociones de raza, negar el racismo y su rearticulación con la desigualdad racial y con el clasismo, facilitando de esta forma que continúen operando, pero de manera sumergida, encubriendo así las relaciones de poder y el orden social racializados. La ideología del mestizaje habría cimentado así, o con estrategias similares, el terreno para deslegitimar la movilización política, social y académica alrededor del tema racial en México (y en el contexto latinoamericano). Este mestizaje ideológico, además, está basado en la enraizada experiencia colonial, en políticas civilizatorias y de modernización y en un racismo científico cruel que está siempre listo para aparecer en escena para negar la existencia y los efectos del racismo en el país (Moreno, 2016: 92).

El racismo "no viene al caso, aquí todos somos mestizos", formas de argumentar que niegan la existencia del racismo y que reducen el problema a la clase social, culpando al sujeto racializado de la desigualdad que experimenta y de su marginación social:

En México no tenemos problemas de racismo, aquí todos somos mestizos y no hay negros como en Colombia (Académico de la Universidad de Guanajuato)

Maldito negro que solo sabe estar jodiendo (Académica mestiza de la Universidad de Guanajuato de origen extranjero).

¡Sí! Mi padre es mixteco y mi madre es zapoteca, así que yo soy mixteco zapoteco, con gran influencia de la cultura, de la lengua y de las prácticas sociales y culturales; aunque podría ser asignado como mestizo; pero tenemos, bueno, yo tengo una raíz indígena muy fuerte (Académico de la Universidad de Guanajuato).

\footnotetext{
${ }^{9}$ El término "post-racial" fue acuñado por el The New York Times el 5 de octubre de 1971, para nombrar el hecho de que políticos y académicos estadounidenses consideraban que la región integrada por Durham y Carolina del Norte había entrado en una era en la que las relaciones raciales pasarían a un segundo plano, dado que pronto serían subsumidas o reemplazadas por otro tipo de relaciones sociales, donde las preocupaciones se centrarían en el incremento de la población, el desarrollo industrial y las fluctuaciones económicas antes que estar atravesadas por el racismo y la discriminación étnica/racial.https://www.nytimes.com/1971/10/05/archives/compactset-up-for-postracial-south.html
} 
¡No!... No..., nunca lo he pensado (la pertenencia étnica). No... (soy) mexicano. (Académico de la Universidad de Guanajuato)

Tales testimonios, que responden a la pregunta sobre la adscripción étnico/racial y sobre discriminación en el espacio de trabajo, como lo observamos en las viñetas, ya han recorrido un arduo entramado y hecho una buena labor deslegitimando la existencia y la importancia del racismo, aún en contextos universitarios y de producción de conocimiento de las ciencias sociales y de las humanidades, siendo adoptados por algunos académicos y políticos en México, que ven a este país consciente o inconscientemente como una sociedad posracial, argumentando que la discriminación basada en el tono de piel es cosa del pasado; por tanto, dudan de la veracidad de los resultados aportados por el INEGI, por OXFAMy por otros estudios que demuestran lo contrario(Zizumbo-Colunga y Flores, 2017:1). Estos estudios evidencian claramente que la desigualdad social y la discriminación en México se basan en la pigmentación de la piel y en la identidad cultural, colocando a los individuos que fenotípicamente poseen tonos de piel más claros y que no ostentan una identidad cultural indígena en la posición de los privilegiados.

¡No!...No... No soy parte de alguna comunidad, minoría, ni nada. (Académico de la Universidad de Guanajuato).

¡No!, soy mestizo (Académico de la Universidad de Guanajuato).

¡Eh!, pues... raza blanca (Académica de la Universidad de Guanajuato de origen extranjero)

El problema, como lo señalan Moreno (2016), Zizumbo-Colunga y Flores (2017), Hurtado (2018) y Solís, Güémez y Lorenzo (2019), es que hablar de racismo es tabú en una sociedad racista y clasista, que sustenta en la ideología del mestizaje la posición social, o es un sacrilegio en la sociedad y en la academia posracial. No obstante, en ambos casos persiste la negación de los procesos de racialización e inclusión segmentada de los percibidos como diferentes y,a diferencia de los entrevistados y entrevistadas de nacionalidad extranjera, preguntar a las y los de origen mexicano sobre su autoadscripción o pertenencia étnica/racial resulta una pregunta extraña, incómoda o impertinente. En estos casos, la respuesta a la pregunta sobre sus identidades o adscripción étnica/racial no procede, porque presumen que la posición sociolaboral de profesor/profesora o de académico/académica e investigador/investigadora con plaza de tiempo completo es resultado del esfuerzo, y que la pigmentación y/o el sexo-género aparentemente no tiene ninguna incidencia en la posición y distribución sociolaboral desigual e inequitativa, del personal administrativo y de los profesionales académicos en esta institución.

"Dígame doctor (o doctora), porque mi trabajo me ha costado". No sea igualado o igualada; aquí todos somos mexicanos; no somos indígenas... no hablamos una lengua indígena; Usted no es negra es morenita... son eufemismos comúnmente empleados para 
no hablar, evadir o no mencionar la condición racial, para situar el problema de la discriminación o el de la escasez de movilidad laboral ascendente (por ejemplo proceso de recategorización para la promoción docente no se les reconoce a todo y todas en igualdad de condiciones, en algunos casos los documentos e información archivada en los expedientes que ofrecen los docentes desaparecen, las demandas por estos aspecto no prosperan, las decisiones que desde la Defensoría de los Derechos Humanos en el Entorno Universitario se toman no son vinculantes, entre otras situaciones similares) al interior de la Universidad de Guanajuato, lo cual perjudica a las personas consideradas políticamente incorrectas, afectando en especial a las personas con fenotipos o colores de piel más oscuros o negras, a las personas que son identificadas como tal y como indígenas de acuerdo con la política de la apariencia, lo que redunda en situaciones de exclusión, rechazo y autoaislamiento.

Todas las oportunidades son para aquellos que nacen con el color de piel que les da beneficios, y/o que provienen de familias que han podido acumular ventajas y transmitirlas, gracias a la falta de pigmentación de su piel y a sus atributos culturales mestizados. Pero existe el otro lado, el de las personas a las cuales en este país no se les da siquiera la oportunidad, pues se les juzga por su supuesta falta de atributos por poseer alguna o varias de estas tres dimensiones: la condición de hablar una lengua indígena, identificarse dentro de una comunidad indígena, negra o mulata, o tener el color de piel más oscuro (Solís, Güémez y Lorenzo, 2019:3).Béjar Navarro (1969), citado por Moreno (2016:102), da cuenta de esta situación al entrelazar conceptualmente las nociones de prejuicio y discriminación racial. Este autor, establece que el prejuicio remite a los rasgos étnicos/raciales estereotipados más visibles y de inmediato opera la discriminación, al otorgar un tratamiento desfavorable a las personas de una cierta categoría étnica/racial, tomando como puntos de referencia aspectos completamente arbitrarios, para mantener una distancia social hacia ellas, por medio de un conjunto de prácticas, más o menos institucionalizadas y racionalizadas.

Como mujer negra... ese enfrentamiento con el primer mundo... ha sido, fue y sigue siendo un encontronazo, empezando con el lenguaje, o con la forma de hablar...(Académica de la Universidad de Guanajuato).

¡Y tú porqué traes el pelo así!, ¡y si te lo peinas...!¡te lo puedo tocar...!¡te lo puedo tocar cuando ya tienen la mano aquí! (indica enseguida su cabeza y cuero cabelludo)...(Académica de la Universidad de Guanajuato).

Esas intenciones a veces vienen con muy mala vibra... (Académica de la Universidad de Guanajuato).

Es que tú tienes líneas de investigación que luego no tributan en nada... (Académica de la Universidad de Guanajuato).

¡No...!Es que a mí me dijeron que tú venias aquí ayudarme a mí...¿ayudarte a ti en qué...? YYo soy parte del comité como mucha gente... (Académica de la Universidad de Guanajuato). 
En consecuencia, es sobre las personas o grupos subalterizados sobre quienes recae el prejuicio racial y el racismo (Moreno, 2016:102); asimismo, el peso de sus repercusiones los instala en el lugar de los individuos en los que por excelencia se deposita la ansiedad por lo racial: "Eres tú la que te pasas diciendo que eres mujer, extranjera y negra, aquí nadie piensa eso...,"“Usted no es negra es morenita...". En general, se percibe cómo en los discursos políticos, académicos y disciplinares que acontecen en la Universidad de Guanajuato, como en otros ámbitos, circulan ideas complejas, confusas o erróneas que tratan de justificar o encubrir el racismo que experimentan los afectados. Castellanos Guerrero resalta que el prejuicio racial, de género, clase, nacionalidad y demás diferencias engendran, con frecuencia, una apreciación negativa consciente o inconsciente de parte de la persona que ejerce dichos prejuicios, de la amenaza constante que su prejuicio representa y puede constituir en la vida laboral y personal de la persona en quien recae. Sin embargo, reconoce que la paradoja está en que, para mantener y reproducir el poder, hay que legitimar las relaciones de explotación y dominación, excluyendo al Otro de la competencia por el hecho de ser diferente o empleando su diferencia como excusa para conseguir eliminarla de dicha competencia(1994: 118-119).

"No sea igualada". La preferimos calladita porque se ve más bonita

En septiembre de 2020, Teodora Hurtado Saa publica la columna titulada "Desigualdad de posiciones, miradas a los patrones de inequidad y discriminación por colores de piel". La autora concuerda con la teoría de la desigualdad de François Dubet (2012 y 2015),quien, desde una mirada crítica, argumenta que preferimos la desigualdad, aunque digamos lo contrario. Al referirse a los ideales y valores de la sociedad francesa ${ }^{10}$, Dubet concibe que, como sociedades contemporáneas, la lógica instrumental, neoliberal y capitalista que nos rige ha contribuido a la ruptura de los lazos de solidaridad y hermandad, que nos impulsaban a desear la igualdad, incluso entre aquellos que no conocíamos. De modo que, reforzamos los desequilibrios y las hostilidades antes que contribuir a la resolución de los problemas de desigualdad social, económica, política y demás.

Por consiguiente, preferir la desigualdad, aunque digamos lo contrario, representa las tensiones y las contradicciones que existen entre la configuración de la igualdad y su contraparte la inequidad, que tradicionalmente han reproducido un orden jerarquizado, genérico, racializado, sexualizado y de clase que sitúa a los individuos en posiciones diferentes, pero sobre todo de poderosos y dominados; así, antes que reducir ciertas

10 De acuerdo con algunas organizaciones civiles de origen francés Liberté, Égalité y Fraternité (libertad, igualdad, fraternidad) son valores fundamentales que definen a la sociedad francesa y a la vida democrática en general. La libertad, o el derecho a vivir libremente y sin opresión o restricciones indebidas por parte de las autoridades, es un valor fundamental en una sociedad democrática. También lo es la igualdad, la cual no se trata únicamente de relacionarse como iguales, sino de que cada persona sea considerada igual ante la ley. La fraternidad, por su parte, se refiere a que todos debemos ser amables y solidarios con los demás.

https://www.liberties.eu/es/about/organisation 
injusticias sociales, las hemos intensificado (Dubet, 2012: 42-43), aunque evocamos la ficción de vivir en sociedades y contar con organizaciones e instituciones democráticas, igualitarias y meritocráticas.

Desde esta noción, las posiciones ventajosas y las oportunidades serán otorgadas prioritariamente a aquellos a quienes se les inscribe como merecedores del "éxito" o de perseguir los mismos ideales e intereses del grupo en las posiciones de poder, es decir dignos de contar con oportunidades y posiciones se hayan esforzado o no para obtenerlas; por tanto, de acuerdo con Dubet (2015: 33), estas personas podrán incrementar y concretar las posibilidades de ensanchar su patrimonio, mientras que los excluidos experimentan mayor precarización de sus condiciones de vida y de trabajo, pisos pegajosos y techos de cristal.

Para quienes nos situamos en un punto interesante o de forma única y destacada nos ubicamos en la esfera de los subalterizados, en especial en el punto focal donde varios sistemas de poder y dominación se unen: género, raza/etnia, clase y ocupación, por ejemplo (como negros/negras o afrodescendientes, como indígenas y pobres), y logramos escalar en el orden social, el mérito más que encontrarse en el hecho de poseer cualidades extraordinarias, que nos permitan romper el techo de cristal y desprendernos relativamente del piso pegajoso, se encuentra en el hecho de emplear estas mismas cualidades para rasgar relativamente las estructuras de opresiones y acceder, moderadamente, tanto a las oportunidades como a las posiciones intermedias o más elevadas (Hurtado, 2020: 1-2), aun cuando, como lo establece Dubet (2015: 35), no se nos considere merecedores de ellas, y sin que al hacerlo necesariamente se pongan en entredicho el orden jerárquico.

En la vida cotidiana, cuando no se brindan los recursos ni se legitima el derecho a la igualdad de oportunidades como de posiciones, independientemente de los estereotipos y prejuicios en torno al género, raza/etnia, clase y ocupación, se sustenta la creencia de que gran parte de las desigualdades que experimentan estos grupos de población son justas y justificables; en otros términos, se sostiene como argumento que las víctimas son responsables de las discriminaciones y exclusiones de las que son objetos, en tanto no se hagan responsables de lo que les pasa. No obstante, cuando se es políticamente incorrecto, cuando no se defienden o profesan los intereses e ideología de los grupos de poder, cuando se es parte de las minorías raciales o de las mayorías desposeídas, las desigualdades de oportunidades y de posiciones son aceptables, porque cada uno merece el lugar que ocupa: culpar a las víctimas, como lo argumenta Dubet. "Los pobres lo son porque no les gusta trabajar", "las mujeres no cuentan con la fortaleza para desenvolverse en cargos de poder" y "las personas negras no disponen ni de la inteligencia ni la "presencia" para ser contratadas" o ser consideradas como miembros prestigiosos de la comunidad académica universitaria". Ello muestra que lo que acontece en los espacios universitarios como la Universidad de Guanajuato, con las poblaciones a las que hacemos referencia en este artículo, se encuadra dentro de las estructuras de 
poder y dominación de las que las instituciones u organizaciones universitarias no están exentas.

\section{A manera de conclusiones}

Los estudios en torno a la naturaleza y a la condición actual del trabajo, la construcción social de las ocupaciones y de los sujetos laborales recientemente se han ocupado por evaluar la precarización paulatina de los empleos, tomando como referencia el contexto universitario y la centralidad que, tanto la labor de investigación como la formación de capital humano, tienen en la vida de los sujetos y en el desarrollo de una sociedad. Estas investigaciones están demostrando cómo el debilitamiento de las condiciones de empleo también ha incidido en la posición sociolaboral de los trabajadores y de las trabajadoras del ámbito académico, ahora cada vez más disímil, dado que están atravesados por aspectos individuales como las características sociodemográficas, y corporativas, como el tipo de contratación y de remuneración, el acceso a los recursos y el reconocimiento institucional, apoyado en estructuras de relaciones de poder.

Sin embargo, los estudios socio-antropológicos contemporáneos en sus inicios abordaban las categorías de clase, género y raza en forma separada; siendo en épocas recientes que resurge la preocupación de las ciencias sociales latinoamericanas por las interacciones entre dichas categorías, en consonancia con la persistencia de la "colonialidad del poder, del saber, del ser y de género", entendiendo que estos modernos patrones de poder se fundan en la imposición de una clasificación racial y étnica jerarquizada de la población. Una estructura de dominación y explotación donde el control de los recursos de producción, de los cuerpos y del trabajo de una población determinada lo detenta otra de diferente identidad de género y color de piel, sustentada en la creencia de la superioridad racial, genérica, epistémica e intelectual.

De igual forma, las contribuciones epistemológicas del Black Feminism, o feminismo de color y del feminismo decolonial, destacan la imposibilidad de separar los mecanismos de opresión de género, raza y clase de otras formas de dominación que padecen las mujeres. Estas corrientes de pensamiento han contribuido al entendimiento de las realidades que atraviesan a los sujetos racializados, siendo categorías útiles también para develar lo que acontece en los contextos universitarios, en particular para entender y explicar las realidades que se circunscriben al ámbito institucional de la Universidad de Guanajuato. Asimismo, me permiten dar cuenta, desde la autoetnografía como metodología narrativa y testimonial, de la centralidad del trabajo en el mundo de vida y determinar cómo ciertas relaciones sociales dominan más cuando funcionan imbricadas, al mismo tiempo, como relaciones de poder, de producción y de estatus social (Alonso, 2004); es decir, como marco y soporte del proceso de apropiación material e inmaterial de la naturaleza y de conocimiento, generando que las conexiones entre relaciones sociales de género, clase, raza y relaciones sociales de producción material e intelectual también sean indisolubles. Ello provoca que el trabajo, la investigación y 
quienes lo realizan sean valorados desde su vinculación con el capital y la ciencia, como desde la posición que ocupan en la relación producción/trabajo y en un orden social racializado.

Por su condición de género y de clase, aunado a sus características étnicas/raciales subalterizadas, las mujeres en general y negras o indígenas en particular han estado marginalmente incluidas a los procesos de producción material e intelectual. Para ellas el trabajo como la producción de conocimiento tiene doble connotación, a la vez que instrumento para su dominación es medio para su emancipación y producción de epistemologías alternas o en resistencia, con las cuales enfrentar el racismo y el sexismo desde el entorno universitario. Es por ello que, pese a que continúan manteniendo una posición subordinada en diversos ámbitos sociales, económicos, políticos y culturales, incluyendo el contexto académico o universitario, es gracias al trabajo que las mujeres, en general, y racializadas, en particular,hemos podido romper algunas de las barreras que limitan el derecho a la equidad, como a la movilidad laboral y a la construcción de nuevas epistemologías.

\section{Bibliografía}

Alonso, L. E. (2004). La sociedad del trabajo: debates actuales. Reis, (107), pp.21-48. http://www.reis.cis.es/REIS/PDF/REIS 107 051168249604623.pdf

Béjar Navarro, R. (1969). Prejuicio y discriminación racial en México. Revista Mexicana de Sociología $31(2) \quad$ pp. 417433.http://revistamexicanadesociologia.unam.mx/index.php/rms/article/view/58 $\underline{440 / 51646}$

Castellanos, A. (1994). Asimilación y diferenciación de los indios en México. Estudios Sociológicos.12(34), pp.101-119. https://estudiossociologicos.colmex.mx/index.php/es/article/view/1000/1000

Castells, M. (2002). Tecnologías de la información y la comunicación y desarrollo global.Revista de Economía Mundial, (7), pp.91107.http://rabida.uhu.es/dspace/handle/10272/422

Castillo, J. J., Moré, P. (2016). Por una sociología del trabajo académico: la precarización del trabajo de enseñar e investigar en la Universidad. Sociología del Trabajo, (88)

pp.7-26. https://revistas.ucm.es/index.php/STRA/article/view/59679/4564456546861 
Castro-Gómez, S.,Grosfoguel, R. (2007). Prólogo. Giro decolonial, teoría crítica y pensamiento heterárquico. EnCastro-Gómez, S., Grosfoguel, R. (comp.) El giro decolonial: reflexiones para una diversidad epistémica más allá del capitalismo global, Siglo del Hombre Editores; Universidad Central, Instituto de Estudios Sociales Contemporáneos y Pontificia Universidad Javeriana, Instituto

Pensar, Bogotá,http://www.unsa.edu.ar/histocat/hamoderna/grosfoguelcastrogomez.pd $\underline{f}$

Da Costa, A. (2014). "Confounding Anti-Racism: Mixture, Racial Democracy, and PostRacial Politics in Brazil". CriticalSociology.http://crs.sagepub.com/content/early/2014/01/30/0896920513 $\underline{508663}$

Curiel, O. (2015). Construyendo metodologías feministas desde el feminismo decolonial. Otras formas de (re) conocer.Gipuzkoa: UPV / HEGOA / SIMRF.http://www.ram-wan.net/restrepo/documentos/ochy.pdf

Barbieri, T. D. (1993). Sobre la categoría género: una introducción teórico-metodológica. Debates En Sociología, (18), pp.145-169. https://revistas.pucp.edu.pe/index.php/debatesensociologia/article/view/6680ht tps://revistas.pucp.edu.pe/index.php/debatesensociologia/article/view/6680/67 84

De Sousa Santos, B. (2018). Introducción a las Epistemologías del Sur. Meneses, M. P.,Bidaseca, Karina Andrea (cord.)Epistemologías del Sur.Ciudad Autónoma de Buenos Aires: CLACSO; Coímbra: Centro de EstudosSociais - CES. Libro digital, http://biblioteca.clacso.edu.ar/clacso/se/20181124092336/Epistemologias_del_ sur_2018.pdf

Dubet, F. (2012). Los límites de la igualdad de oportunidades. Nueva Sociedad(239). pp.42-50. https://halshs.archives-ouvertes.fr/halshs-00718737/document

Dubet, F. (2015), ¿Por qué preferimos la desigualdad? (aunque digamos lo contrario). 
Revista Punto Género N ․ 16 Diciembre de 2021

ISSN 2735-7473 / 51- 78

Editorial: Siglo Veintiuno.

Ellis, C., Adams, T. E.,Bochner, A. P. (2019).Autoetnografía: un panorama. En Bénard Calva, S. M. (Selección de textos) Autoetnografía. Una metodología cualitativa. .Universidad Autónoma de Aguascalientes, El Colegio de San Luis, A.C. https://editorial.uaa.mx/docs/autoetnografia2.pdf

García Ruiz, P. (1999).El laberinto social. Cuestiones básicas de sociología. Ediciones Universidad de Navarra S.A. Pamplona.

Gutiérrez Garza, E., González G. E. (2010). De las teorías del desarrollo al desarrollo sostenible. México: Siglo XIX Editores. Universidad Autónoma de Nuevo León.

Hartman, S. V. (2007).Lose yourmother: A journey a longthe Atlantic Slave Route, Farrar, Straus and Giroux, Nueva York.

Hill Collins, P. (2000).Black feminist thought: knowledge, consciousness and the politics of empowerment, Nueva York: Routledge.

Hurtado Saa, T. (2011).Mujeres, negras e inmigrantes construyendo la ocupación de "proveedoras" de servicios afectivos y "vendedoras" de bienes eróticoamorosos en los espacios transnacionales(tesis doctoral). Universidad Autónoma Metropolitana - Unidad Izatapalapa

Hurtado Saa, T. (2016). Interseccionalidad de género, etnia/raza, ocupación y nacionalidad en la producción académica de Universidad de Guanajuato, en Góngora Cervantes V., Vázquez Valenzuela,R. (coordinadoras),Perspectiva de género en la práctica educativa de la Universidad de Guanajuato. Aproximaciones Feministas.Universidad de Guanajuato, Gto. 2016.

Hurtado Saa, T. (2018). Racismo institucional y epistémico desde un enfoque interserseccional: ser mujer, extranjera y negra en la producción académica en México.En OcoróLoangoA., Alves Cordeiro,M. J. J. (coordinadoras) Negritudes e africanidades na América Latina e no Caribe. Volumen, Editorial ORGs: Ribeirão Gráfica e Editora, 2018: 53-71. 
Hurtado Saa, T. (2020). Desigualdad de oportunidades y/o desigualdad de posiciones, miradas a los patrones de inequidad y discriminación por colores de piel.Revista http://dcimarron.org/images/dcimarro10/THEODORA18.pdf

D'CIMARRÓN(14).

Kergoat, D. (2003). De la relación social de sexo al sujeto sexuadoll, Revista Mexicana de Sociología65(4) pp.841-861.

Lander, E. (Comp. Editor). (2000). La colonialidad del saber: eurocentrismo y ciencias sociales. Perspectivas latinoamericanas. Consejo Latinoamericano de Ciencias Sociales (CLACSO), Buenos Aires. http://biblioteca.clacso.edu.ar/clacso/sur-sur/20100708034410/lander.pdf

Llomovatte, S. y Wischnevsky, J. (1998). Condiciones laborales de los docentes universitarios en los 90. Algunos resultados de investigación. Praxis educativa $3(3)$, pp.1526.https://cerac.unlpam.edu.ar/index.php/praxis/article/viewFile/193/181

Lugones, M. (2008). Colonialidad y género. TabulaRasa (9). pp.73-101. https://www.revistatabularasa.org/numero-9/05lugones.pdf

Mbembe, A.(2011[2006]), Necropolítica, editorial Melusina.

Méda, D. (1996). "El valor trabajo visto en perspectiva". Revista Internacional del Trabajo,(115:6) pp.686-700.

Méda, D. (2007). “¿Qué sabemos sobre el trabajo?”. Revista de Trabajo, 3(4) pp. http://www.trabajo.gob.ar/downloads/estadisticas/2007n04 revistaDeTrabajo.p $\underline{\mathrm{df}}$

Mignolo, W. (2000). La colonialidad a lo largo y a lo ancho: el hemisferio occidental en el horizonte colonial de la modernidad.La colonialidad del saber: eurocentrismo y ciencias sociales. Lander E. (comp.). Perspectivas latinoamericanas. Buenos Aires, 
Revista Punto Género N ․ 16 Diciembre de 2021

ISSN 2735-7473 / 51- 78

https://www.tni.org/files/download/La\%20colonialidad\%20del\%20saber.\%20Eu rocentrismo\%20y\%20ciencias\%20sociales.pdf

Mohanty, C. T. (2008 [1986]). Bajo los ojos de Occidente. Academia feminista y discurso colonial. Descolonizar el Feminismo: Teorías y Prácticas desde los Márgenes. Hernández Castillo R. A. y Suárez Navaz, L. (coord.). España, Cátedra.

Moreno Figueroa, M. G. (2016) El archivo del estudio del racismo en México. RevistaDesacato pp.92-107).

http://www.scielo.org.mx/scielo.php?script=sci arttext\&pid=S1607$\underline{050 \times 2016000200092}$

Neffa, J. C. (2003). Grandes rasgos de la evolución del concepto de trabajo desde la antigüedad hasta fines del XIX en López, M. (2009). La evolución del concepto del trabajo a lo largo de la historia. Recuperado de https://es.slideshare.net/Laboralyorganizacional/significacion-del-trabajohumano-neffa

Neffa, J. C. (2010). La transición desde los — verdaderos empleosll al trabajo precario.En De la Garza Toledo, E., Neffa, J. C. (coord.) Trabajo, identidad y acción colectiva.Ed. CLACSO, Universidad Autónoma Metropolitana-Iztapalapa, Plaza y Valdés, S.A. de C.V. Madrid: 43-80pp.

Nussbaum, M. (2012). Crear capacidades. Propuesta para el desarrollo humano. España: Paidós

Parella, R. S. (2005). La segregación laboral y vulnerabilidad social de la mujer inmigrante a partir de la interacción entre clase social, género y etnia. En Solé. C., Flaquer. L. (Eds.) Uso de las políticas sociales por las mujeres inmigrantes, Instituto de la Mujer (Ministerio de Trabajo y Asuntos Sociales), Madrid: 95$136 p$.

Parsons, T. (2013). El sistema social.https://teoriasuno.files.wordpress.com/2013/08/elsistema-social-talcott-parsons.pdf 
Revista Punto Género N. 16 Diciembre de 2021

ISSN 2735-7473 / 51- 78

Pfefferkorn, R. (2007). El trabajo, un eje fundamental de las relaciones sociales de sexo, en Revista Ciencias Sociales. lquique(18), pp.5370.https://www.redalyc.org/pdf/708/70801804.pdf

Quijano, A. (2000).Colonialidad del poder, eurocentrismo y América Latina. En Lander E. (comp.) La colonialidad del saber: eurocentrismo y ciencias sociales. Perspectivas latinoamericanas. Buenos Aires, CLACSO, pp.201-246. https://www.tni.org/files/download/La\%20colonialidad\%20del\%20saber.\%20Eu rocentrismo\%20y\%20ciencias\%20sociales.pdf

Rodríguez Reyes, A. (2016). El giro decolonial en el siglo XXI, Revista Ensayos Pedagógicos.XI(2) pp.33-158. https://www.revistas.una.ac.cr/index.php/ensayospedagogicos/article/view/915 $\underline{0 / 10675}$

Scott, J. W. (1986). Gender: A Useful Category of Historical Analysis, The American Historical Review. 91 (5)pp.1053-075.

Sen A. (2000).Desarrollo y libertad.Ed.Planeta. Barcelona.

Sennett, R. (2000). La corrosión del carácter: las consecuencias personales del trabajo en el nuevo capitalismo.Barcelona: Anagrama......

SpivaK, G.(2009 [1999])¿Pueden hablar los subalterizados? Edición Museud'ArtContemporani de Barcelona. https://es.scribd.com/document/364514133/spivak-pueden-hablar-lossubalternos-1-pdf

Solís, P., Braulio, G., Lorenzo Holm,V. (2019).El impacto de las características étnicoraciales en la desigualdad de oportunidades en México (Resumen de Investigación), OXFAM México. https://www.oxfammexico.org/sites/default/files/Por\%20mi\%20raza\%20hablara \%20la\%20desigualdad $0 . p d f$

Vásquez Rocca, A. (2017).Byung-Chul Han: la sociedad de la transparencia, autoexplotación neoliberal y psicopolítica. De lo viral-inmunológico a lo 
Revista Punto Género N ․ 16 Diciembre de 2021

ISSN 2735-7473 / 51- 78

neuronal-estresante.Nómadas Revista Crítica de Ciencias Sociales y Jurídicas.52(4)

pp.1-24.

https://www.researchgate.net/publication/320721069 Byung-

Chul Han la sociedad de la transparencia autoexplotacion neoliberal y $p$ sicopolitica De lo viral-inmunologico a lo neuronal-estresante

Wade, P., Urrea Giraldo, F. Viveros Vigoya,M. (2008). Debates contemporáneos sobre raza, etnicidad, género y sexualidad en las ciencias sociales. En(eds.) Wade P., Urrea F., Viveros Mara. Raza, etnicidad y sexualidades. Ciudadanía y Multiculturalismo en América Latina,Universidad Nacional de Colombia-Centro de Estudios Sociales, Universidad del Valle y Universidad de Manchester, Bogotá: 41- 66pp.

Zizumbo-Colunga, Da., Martínez,I. F. (2017).¿Es México un país post-racial?La desigualdad y el tono de piel en las Américas, Centro de Investigación y Docencia Económicas. pp. 1 11https://www.vanderbilt.edu/lapop/insights/ITB031es.pdf 